\title{
Multimodal Particle-Size Distribution or Fractal Surface of Acrylic Acid Copolymer Nanoparticles: a Small-Angle X-ray Scattering Study Using Direct Fourier and Indirect Maximum-Entropy Methods
}

\author{
BY JÜRGEN J. MÜLLER* \\ Max-Delbrück-Center for Molecular Medicine, Robert-Rössle-Strasse 10, D-13122 Berlin, Germany \\ STEEN HANSEN \\ Department of Mathematics and Physics, Royal Veterinary and Agricultural University, Thorvaldsensvej 40, \\ 1871 FRB C, Denmark \\ GEROLD LUKOWSKI \\ Department of Pharmaceutics, E.-M.-Arndt-University, Jahnstrasse 17, D-17489 Greifswald, Germany \\ AND KLAUS GAST \\ Max-Delbrück-Center for Molecular Medicine, Robert-Rössle-Strasse 10, D-13122 Berlin, Germany
}

(Received 3 March 1995; accepted 31 May 1995)

\begin{abstract}
Acrylic acid copolymers are potential carriers for drug delivery. The surface, surface rugosity and the absolute dimension of the particles are parameters that determine the binding of drugs or detergents, diffusion phenomena at the surface and the distribution of the carrier within the human body. The particle-size distribution and surface rugosity of the particles have been investigated by smallangle $\mathrm{X}$-ray scattering and dynamic light scattering. Direct Fourier transform as well as a new strategy for the indirect maximum-entropy method MAXENT are used for data evaluation. Scattering equivalence of a pure multimodal distribution of hard spheres (five populations) and a mixed multimodal-surface-fractal model (four populations) was found. Model calculations and dynamic lightscatteing experiments gave evidence of the multimodal particle-size distribution combined with the fractal surface of the carrier. The main moiety consists of particles $90 \mathrm{~nm}$ in diameter which are surface fractals in the $10 \mathrm{~nm}$ region.
\end{abstract}

\section{Introduction}

Acrylic acid copolymers with dimensions of about $100 \mathrm{~nm}$ are potential carriers for drug delivery (Verdun, Couvreur, Vranckx, Lenaerts \& Roland, 1986; Tarcha,

* To whom all correspondence should be addressed.

(C) 1995 International Union of Crystallography

Printed in Great Britain - all rights reserved
1991). The surface, surface rugosity and the absolute dimensions or the size distribution of the particles are parameters that determine the binding of drugs or detergents, diffusion phenomena at the surface, the recognition and clearance by the reticuloendothelial system and the distribution of the carrier within the body (Müller, 1991, Kreuter, 1983; Pfeifer, 1987). Therefore, these structure parameters must be accurately known to permit a correct functional interpretation. The determination of the size distribution from small-angle $\mathrm{X}$-ray scattering data is an underdetermined problem and consequently there are many possible solutions that can fit the experimental data adequately.

When using indirect methods, some additional constraints must be introduced for selection of a single solution from the set of possible solutions. Various constraints have been tried for the analogous problem of determination of the distance distribution function from small-angle scattering data: Glatter (1977) uses a smoothness constraint (choosing the 'smoothest' of the possible solutions), Moore (1980) prefers decomposition of the distribution into a special functional system with appropriate properties, Hansen \& Pedersen (1991) propose the maximum-entropy method and Svergun (1992) uses a combination of more subjective criteria for selection of the 'best' distribution. Constraints can be taken into account in a similar way for the determination of the particle-size-distribution function (e.g. Glatter, 1980; Potton, Daniell \& Rainford, 1988; Svergun, Semenyuk \& Feigin, 1988). However, it has to be noted 
that the problem of the determination of the sizedistribution function from small-angle scattering data is usually even more ill conditioned than the determination of the distance-distribution function (this will be apparent from a singular-value decomposition of the transformation matrix which takes the distribution to be estimated from real space into reciprocal space). Which method of regularization should be applied depends on the prior knowledge of the scatterer and the data obtained. For example, for low-resolution data, a smoothness constraint is probably appropriate, whereas the maximum-entropy method might be appropriate for data of higher resolution or when some prior information about the shape of the scatterer (e.g. from other experiments) can be included in the analysis (Müller \& Hansen, 1994).

In the present paper, we have used the program MAXENT (Müller and Hansen, 1994) for the determination of particle-size distributions. We will show by a simulation that a good prior estimate for the particle-size distribution has to be chosen to obtain reliable results and to avoid artefacts.

For the data presented here, direct methods of data evaluation gave evidence of a multimodal particle-size distribution combined with a fratal surface of the particles. By MAXENT, an additional particle population is detected, but model calculations in combination with experimental results of dynamic light scattering and hints from electron microscopy give evidence that surface rugosity is more probable than an additional population of small particles or inner-electron-density fluctuations at the given structure resolution.

\section{Experimental}

\subsection{Small-angle $X$-ray scattering ( $S A X S)$}

The copolymer particles of $14.4 \%$ acrylic acid, $3 \%$ acrylamide, $26.6 \%$ acrylic butyl ester and $56 \%$ methacrylic methyl ester (CAA nanoparticles) were produced by emulsion polymerization (Lukowski, Müller, Müller \& Dittgen, 1992). The samples were suspended in double-distilled water and investigated at room temperature in thin-walled glass capillaries $1 \mathrm{~mm}$ in diameter as described recently (Müller, Lukowski, Kröber, Damaschun \& Dittgen, 1994). A second series was measured in a physiological sodium chloride solution $(0.15 \mathrm{M} \mathrm{NaCl})$ to compensate free charges at the nanoparticle surface.

The measurement of the scattered intensity was done using a Kratky diffractometer (A. Paar, KG, Graz, Austria) with a slit system that guarantees an information loss-free registration for particle dimensions smaller than $260 \mathrm{~nm}$. The upper boundary of the scattering vector was $s_{\max , \exp }=0.6 \mathrm{~nm}^{-1}$, providing a resolution of about $5 \mathrm{~nm}[s=4 \pi \sin (\Theta) / \lambda ; \lambda$ is the wavelength, $2 \Theta$ is the scattering angle]. The scattered $\mathrm{Cu} K \alpha$ radiation was monochromatized by pulse-height discrimination and a
$7 \mu \mathrm{m} \mathrm{Ni}$ filter and then registered by a proportional counter. Diffractometer distortion corrections (slit-length and slit-width desmearing) were done using direct methods (Müller, 1992) as well as the indirect method $M A X E N T$, using real experimental beam profiles. Data evaluation and extrapolations to infinite polymer dilution were carried out for two concentration series $(1.36,2.26$, $4.65,8.9,17.9$ and $39.5 \mathrm{~g} \mathrm{l}^{-1}$ in double distilled water; $4.41,8.57,17.27$ and $34.5 \mathrm{~g} \mathrm{l}^{-1}$ in $0.15 \mathrm{M} \mathrm{NaCl}$ ). The procedures have been described recently for CAA nanoparticles (Müller, Lukowski, Kröber, Damaschun \& Dittgen, 1994). The desmeared scattering curves of the nanoparticles for infinite dilution, resulting from the two desmearing methods, are identical for both solvent conditions within the experimental errors. The scattering curve of particles in distilled water is used for further interpretation in this paper.

\subsection{Dynamic light scattering}

Dynamic light-scattering investigations were performed using a spectrometer that has been described previously (Gast, Damaschun, Misselwitz \& Zirwer, 1992). The measurements were carried out at $293 \mathrm{~K}$, at a scattering angle of $90^{\circ}$ and at the wavelength $632.8 \mathrm{~nm}$ of a helium neon laser operating at $35 \mathrm{~mW}$ output power. The suspensions were filtered through $0.4 \mu \mathrm{m}$ Nuclepore filters directly into $100 \mu \mathrm{l}$ flow-through cells (Hellma, Germany). The homodyne time-autocorrelation functions of the scattered light intensity, $G^{2}(\tau)$, were calculated by a 90 -channel multibit multiple- $\tau$ correlator and then fed into an on-line coupled PC equipped with a transputer board, ALV-800 (ALV Laser-Vertriebsgesellschaft mbH, Germany) for data evaluation using the program $\mathrm{CON}$ TIN (Provencher, 1982).

\section{Theory}

\subsection{Particle-size distribution function - direct method in $X$-ray scattering}

The calculation of the size-distribution function $N(D)$ for spherical particles by a direct method has been described recently by Walter, Gerber \& Kranold (1983) and Müller, Lukowski, Kröber, Damaschun \& Dittgen (1994). To determine $N(D)$ from the scattered intensity equation (1) has to be inverted:

$$
I(s)=\int_{L_{\min }}^{L_{\max }} N(D) I_{D}(s, D) \mathrm{d} D .
$$

$L_{\max }, L_{\min }$ are the diameters of the largest and smallest particles, respectively, in a given sample and detectable with the given diffractometer slit configuration. $I_{D}(s, D)$ is the intensity scattered from a single particle with diameter $D$. For $I_{D}(0, D) \propto V^{2}(D), N(D)$ is a number distribution; $V(D) N(D)$ is the volume-weighted distribution, where $V(D)$ is the volume of a sphere with diameter 
$D . N(D)$ can be calculated from the scattered intensity at discrete points $k \Delta s$ :

$$
\begin{aligned}
N(D)= & \Delta s^{2} / 2 \pi^{2} \sum_{k=0}^{M} k I(k \Delta s)\{\sin (K \Delta s D) \\
& \times\left[8 / D^{5}-4(k \Delta s)^{2} / D^{3}\right] \\
& +\cos (k \Delta s D)\left[(k \Delta s)^{3} / D^{2}\right. \\
& \left.\left.-8 k \Delta s / D^{4}\right]\right\}+R_{i} .
\end{aligned}
$$

$R_{i}$ are analytical correction terms for termination-error reduction when the scattering curve follows a constant $/ s^{3}$ or constant $/ s^{4}$ law at large angles (Müller et al., 1994). The scattered intensity is recalculated from the distribution function by

$$
I_{\mathrm{rec}}(s)=\sum_{i=L_{\min } / \Delta D}^{L_{\max } / \Delta D} N(i \Delta D) I(s, i \Delta x),
$$

where $\Delta D$ is the step width used for diameters.

\subsection{Particle-size distribution function - indirect maxi- mum-entropy method}

The determination of the size distribution from smallangle scattering data is an underdetermined problem and consequently there are many possible solutions.

In general, for problems where many different distributions can fit the data adequately, some principle for choosing a single one is necessary. For this purpose, the maximum-entropy method (MAXENT) can be used (see e.g. Jaynes, 1983). Using MAXENT for selection of a single distribution from many distributions $N=$ $\left(N_{1}, \ldots, N_{N_{\max }}\right)$, which all fit the data, the one is chosen that maximizes the entropy $S$ as given by Skilling (1988):

$$
S(N, m)=\sum_{j=1}^{N_{\max }}-N_{j} \ln \left(N_{j} / m_{j}\right)+N_{j}-m_{j},
$$

where $m=\left(m_{1}, \ldots, m_{N_{\max }}\right)$ is a prior estimate of $N$.

For the present application, the maximization of the entropy is done subject to the constraint that the distribution fits the $M$ data points $I\left(s_{1}\right), \ldots, I\left(s_{M}\right)$. The quality of the fit is given by the usual expression:

$$
\chi^{2}=\sum_{i=1}^{M}\left(I\left(s_{i}\right)-\sum_{j}^{N_{\max }} A_{i j} N_{j}\right)^{2} / \sigma_{i}^{2},
$$

where $\sigma_{i}$ is the standard deviation for the Gaussian noise at a data point $i$ and the matrix $\mathbf{A}$ is given by

$$
\begin{aligned}
A_{i j}= & 9 V\left(D_{j}\right)\left(\left\{\sin \left[s_{i}\left(D_{j} / 2\right)\right]-s_{i}\left(D_{j} / 2\right)\right.\right. \\
& \left.\left.\times \cos \left[s_{i}\left(D_{j} / 2\right)\right]\right\} /\left[s_{i}\left(D_{j} / 2\right)\right]^{3}\right)^{2} \Delta D,
\end{aligned}
$$

where $\Delta D=D_{j+1}-D_{j}$. By this definition of the transformation matrix $\mathbf{A}$, the size distribution that is determined is a volume-weighted distribution.
The simultaneous curve fitting and maximization of the entropy are achieved by solution of the equation.

$$
\nabla\left(\alpha S+\chi^{2}\right)=0,
$$

where $\alpha$ is a Lagrange multiplier allowing the $\chi^{2}$ to obtain a predetermined value. A simple algorithm for this purpose is described by Steenstrup (1985).

In small-angle scattering, the maximum-entropy method has previously been used for estimation of distance distribution functions (Hansen \& Pedersen, 1991; Müller \& Hansen, 1994) and size distributions (e.g. Potton, Daniell \& Rainford, 1988; Morrison, Corcoran \& Lewis, 1992; Jemian \& Allen, 1994).

\subsection{Determination of a fractal dimension}

The scattering of fractals has been described earlier by Pfeifer (1984), Schmidt (1991) and others. Schmidt (1991) has shown that surface fractals scatter as

$$
I(s)=\text { constant } / s^{6-d}
$$

and mass fractals as

$$
I(s)=\text { constant } / s^{d},
$$

assuming constant electron density in the particle matrix.

The fractal dimension $d$ has to be less than 3 . Therefore, if the scattering curve declines more strongly than $s^{-3}$, the sample cannot be a mass fractal but only a surface fractal. $d=2$ characterizes a smooth surface, $d=3$ a strong fractality. The self-similarity region of the fractal is restricted to a real-space dimension of $\pi / s_{\min }>r>\pi / s_{\max }$ with $s_{\min }$ and $s_{\max }$ being the boundaries of the power-law region in the scattering curve. Pfeifer (1984) defined the condition

$$
s_{\max } / s_{\min }>2^{1 / d}
$$

that must be fulfilled to accept the value $d$ as a well defined dimension.

\subsection{Dynamic light scattering (DLS)}

The program package CONTIN (Provencher, 1979, 1982) was used to estimate either a distribution of translational diffusion coefficients $D_{T}$ from which the corresponding hydrodynamic diameters can be calculated via the Einstein equation

$$
D_{T}=K T /(3 \pi \eta D)
$$

or directly the distribution of diameters assuming the particles to be compact spheres ( $K$ is Boltzmann's constant, $T$ is the absolute temperature, $\eta$ is the viscosity). While in the former case the ordinate of the distribution function is proportional to the scattered intensity per size interval $\propto V^{2}(D) N(D)$, a weight concentration distribution $\propto V(D) N(D)$ is generated in the second case (identical with the volume-weighted diameter distribution in SAXS). Thus, the second procedure appears to be 


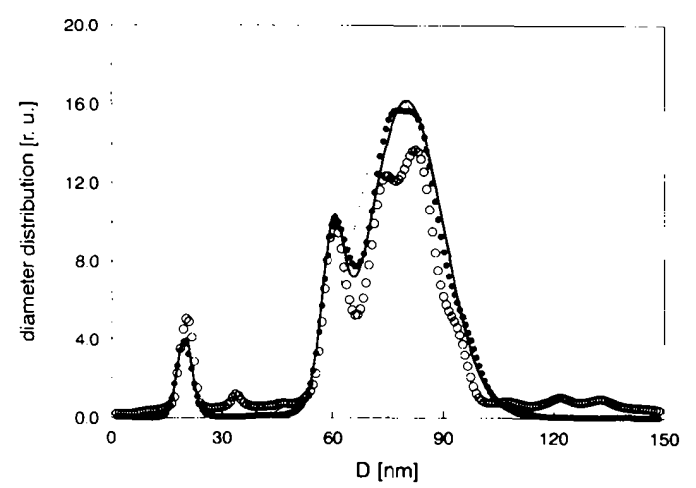

Fig. 1. Influence of the prior on the diameter distribution of spheres calculated by the maximum-entropy method MAXENT. Theoretical distribution calculated from equation (12); $\cdots$ Gaussian prior; ० ० $\circ M A X E N T$ result using a uniform flat prior; $\bullet \bullet-M A X E N T$ result using the Gaussian prior.

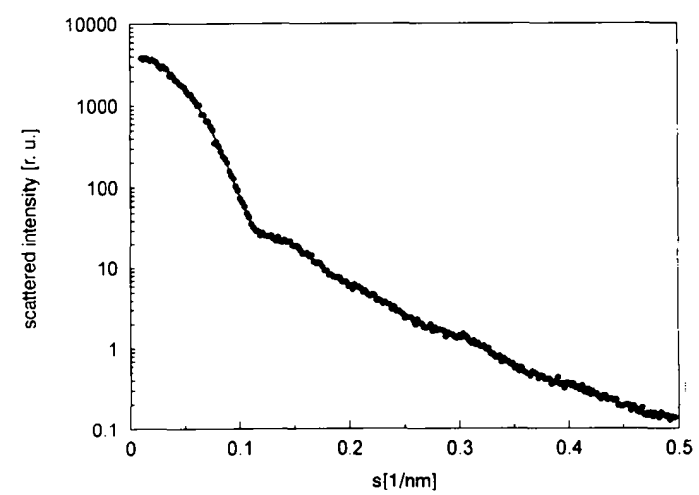

Fig. 2. Scattering curve of a system of spheres with a diameter distribution shown in Fig. 1. $\bullet$ Theoretical scattering curve, $5 \%$ constant relative noise; $-M A X E N T$ fit using a Gaussian prior.

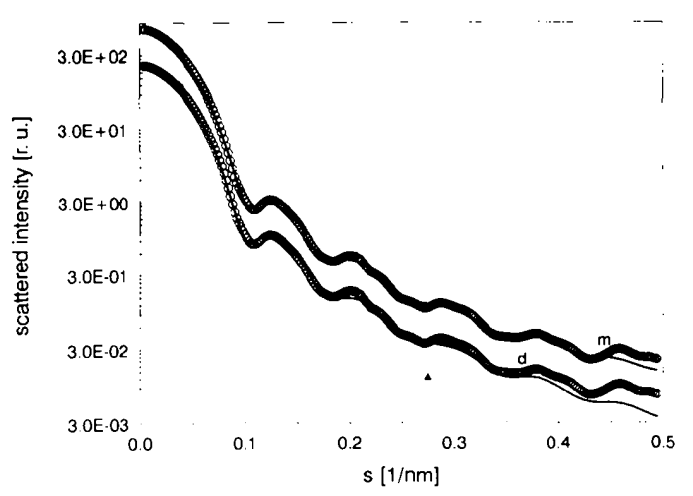

Fig. 3. Comparison of the experimental scattering curve of CAA nanoparticles with model scattering curves. Upper two curves $(m)$ : $\bigcirc 0 \circ$ experiment data; — MAXENT fit using the modified particlesize distribution function (shown in Fig. 4) as prior. Lower two curves $(d)$ : 000 experimental data; — reconstituted scattering curve using the modified distribution (shown in Fig. 4) in equation (3). $\Delta$ marks the point of divergence between the experimental and the reconstituted curves. The upper curves are shifted vertically by an arbitrary value. more useful but it has two disadvantages. Firstly, a proper assumption for the relation between the diameter and particle mass has to be made. Secondly, fast-decaying components in the autocorrelation function are sometimes overestimated. Thus, spurious small-particle components may appear in the diameter-distribution function. We have applied both data-evaluating schemes in order to get reliable results.

\section{Results}

Previous estimates of size distributions using the maximum-entropy method have all used a uniform prior. However, the importance of the prior has been demonstrated for distance distributions in small-angle scattering (Müller \& Hansen, 1994), and similarly the choice of prior is also relevant for estimation of size distributions. A theoretical example demonstrating the importance of the prior is discussed here. The simulated size distribution consists of three Gaussians (Fig. 1):

$$
\begin{aligned}
N(D)= & \exp \left[-0.5(D-20)^{2} / 2^{2}\right] \\
& +2 \exp \left[-0.5(D-60)^{2} / 3^{2}\right] \\
& +4 \exp \left[-0.5(D-80)^{2} / 10^{2}\right]
\end{aligned}
$$

The corresponding scattered intensity is shown in Fig. 2 with $5 \%$ noise added. MAXENT has now been used to estimate the size distribution from the noisy data. Firstly using a uniform prior gives a result with artefacts, most importantly oscillations that give a splitting of the main peak. This splitting is to be expected as the MAXENT estimate is biased towards the uniform prior and the entropy of a solution with two smaller peaks is then higher than that of a solution with one larger peak. Secondly, using a Gaussian of free position, width and height as the prior gives an estimate much closer to the original size distribution. The position, width and height of the prior used for the estimate are chosen as those yielding the solution that has the maximum entropy. For estimation of size distributions, a Gaussian size distribution will usually convey the expectation of the experiment better than a uniform prior as a flat size distribution is not a frequently encountered situation. The MAXENT fit shown in Fig. 2 is that corresponding to the Gaussian prior.

The experimental slit-corrected scattering curve of the CAA nanoparticles at infinite dilution is shown in Fig. 3. Any model fitting with coated spheres or ellipsoids failed (Müller, Lukowski, Kröber, Damaschun \& Dittgen, 1994) but a particle-size distribution function for spheres can be calculated by the direct method (Fig. 4). This distribution shows one main peak and spurious oscillations resulting from the restriction of the measuring interval to $s_{\max , \exp }=0.6 \mathrm{~nm}^{-1}$. The true distribution cannot be negative and the mean of the envelopes of the oscillating curve is used as the more probable diameter 
distribution. The contribution of particles with diameters smaller than $12 \mathrm{~nm}$ may also be an artefact from wrong curve extrapolation to larger angles not measured experimentally. This modified curve $N(D) V(D)$ has been used to synthesize the scattering curve corresponding to (3) with $\Delta D=1 \mathrm{~nm}$. The radii of gyration $R_{G}=38.7$ (9) and $37.8 \mathrm{~nm}$, the weight-averaged volumes $V=$ $3.8(3) \times 10^{5}$ and $4.0 \times 10^{5} \mathrm{~nm}^{3}$ and the largest diameters $L_{\max }=130(5)$ and $126 \mathrm{~nm}$, respectively, are identical for experimental and reconstructed data. Both scattering curves are shown in Fig. 3. Up to $s=0.275 \mathrm{~nm}^{-1}$, the agreement is complete within the error limits. For larger scattering vectors, the experimental scattered intensity is higher than the calculated intensity.

The experimental data are drawn on a doublelogarithmic scale in Fig. 5. Each power-law curve will be a straight line with the inclination of the negative power. An oscillation of the scattering curve around the straight line with an inclination of -3.65 is visible. This

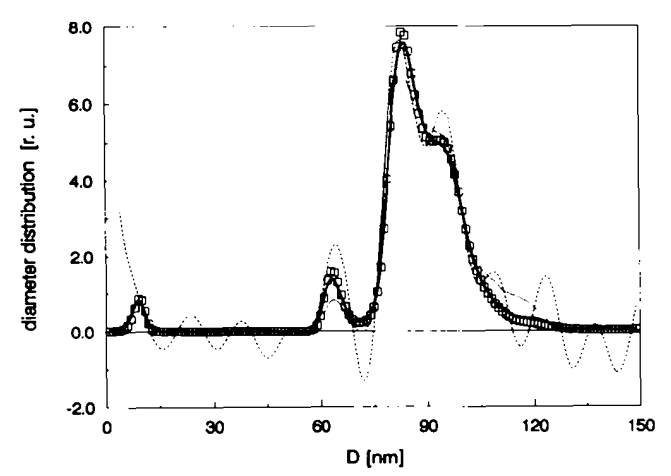

Fig. 4. Diameter distribution functions of CAA nanoparticles determined from X-ray scattering. - - - Calculated with direct method; $\cdots$ modified result of the direct method and prior for $M A X E N T$; MAXENT result using the modified distribution obtained by the direct method as prior; $\square \square \square$ MAXENT result, using a free Gaussian as prior.

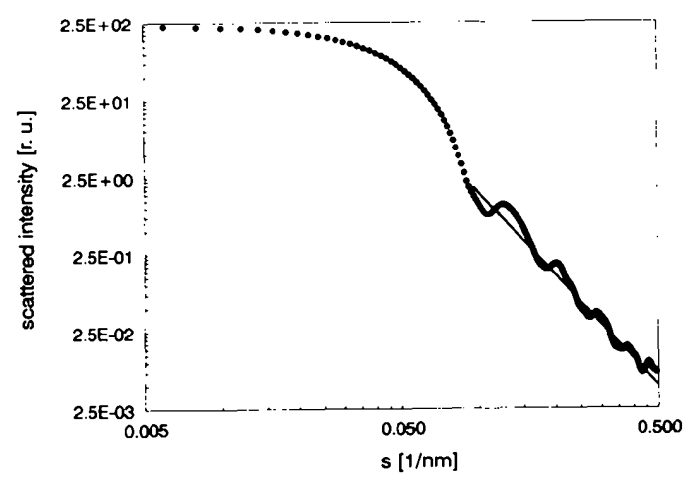

Fig. 5. X-ray scattering curve of CAA nanoparticles on a logarithmic scale for visualization of surface fractality. $\bullet \bullet$ Experimental data; — straight line with an inclination of -3.65 (fractal dimension $d=2.35$ ). deviation from Porod's law (see Glatter \& Kratky, 1982) can be caused by several structural features. Surface rugosity, inner-electron-density fluctuations or a combination of both, or an additional particle moiety with diameter less than $12 \mathrm{~nm}$, which cannot be excluded from the result of the direct data handling as discussed above, can produce the additional intensity detectable in this angular region. For the first possibility, the data evaluation follows $(8)-(10)$ for a fractal sample. If there exists any fractality, it must be a surface fractal because the power has to be smaller than 3 for a mass fractal. The dimension of a surface fractal is then $d=2.35(10)$ as follows from (8). This value characterizes a rather smooth surface. The boundaries of the self-similarity interval are $\pi / 0.41 \mathrm{~nm}(7.7 \mathrm{~nm})$ and $\pi / 0.275 \mathrm{~nm}$ $(11.4 \mathrm{~nm})$, taking the point of divergence between the experimental curve and the reconstituted one and the end of the power-law interval, respectively. Formally, (10) is fulfilled with $s_{\max } / s_{\min }=1.49$ being larger than $2^{1 / d}=$ 1.34 , so that $d=2.35$ can be accepted as a fractal dimension. At smaller angles, the experimental scattering curve oscillates around the $s^{-4}$ curve showing that the particles have smooth surfaces at this resolution.

We used the maximum-entropy method MAXENT to obtain more detailed information from the X-ray scattering. The result of the maximum-entropy search was shown in Fig. 4. If the directly determined modified diameter distribution is used as a prior, the same features as in the prior are visible for diameters $>60 \mathrm{~nm}$ (weighted mean diameter $90 \mathrm{~nm}$ ) but an additional small population with diameters around 5 to $12 \mathrm{~nm}$ is also evident. The scattering curve is fitted on the basis of this distribution up to $s=0.45 \mathrm{~nm}^{-1}$ within the experimental error limits (Fig. 3). This means that no fractality would be necessary to explain the tail of the curve at larger scattering vectors, at least for dimensions larger than $5 \mathrm{~nm}$. Because the results of a maximum-entropy search can be biased in the wrong direction by an inadequate prior as shown above, we used a free Gaussian distribution as a much simpler prior. The MAXENT result is identical with the first one (Fig. 4), showing the independence of the determined particle-size distribution on the used special priors and confirming at least four particle subpopulations with diameters larger than $60 \mathrm{~nm}$ and one with about $10 \mathrm{~nm}$.

Contrast-variation experiments would be necessary to detect inner-electron-density fluctuations, which may also be the reason for the increased scattering at larger angles. Another way to confirm the surface rugosity is the use of additional biophysical methods, which deliver information about the particle structure which is not influenced by inner inhomogeneities. Therefore, dynamic light-scattering (DLS) experiments are suitable. The sizedistribution functions calculated from DLS data are shown in Figs. 6 and 7. The distribution in Fig. 6 $\left[\propto V^{2}(D) N(D)\right]$ represents the relative scattered intensity per size interval and consists mainly of one peak with an 
averaged diameter of $104 \mathrm{~nm}$. A very small second peak contributing only about $1 \%$ to the total scattered intensity can be seen at $41 \mathrm{~nm}$ diameter. The weight distribution function in Fig. $7[\propto V(D) N(D)]$ shows two peaks, having average diameters of 36 and $98 \mathrm{~nm}$, respectively. According to this distribution, the particles of the smaller peak would contribute about $8 \%$ to the total mass. The main result is that the predominant portion of the particles has diameters around $100 \mathrm{~nm}$, which means about $10 \%$ larger than that determined by X-ray scattering. Because the smaller species contributes only very weakly to the total scattered intensity, we cannot unambiguously decide whether it really represents a distinct particle population or the main peak is only skewed towards smaller diameters. The smaller particle population is not well defined by DLS. Furthermore, there is no evidence for a population with diameters of

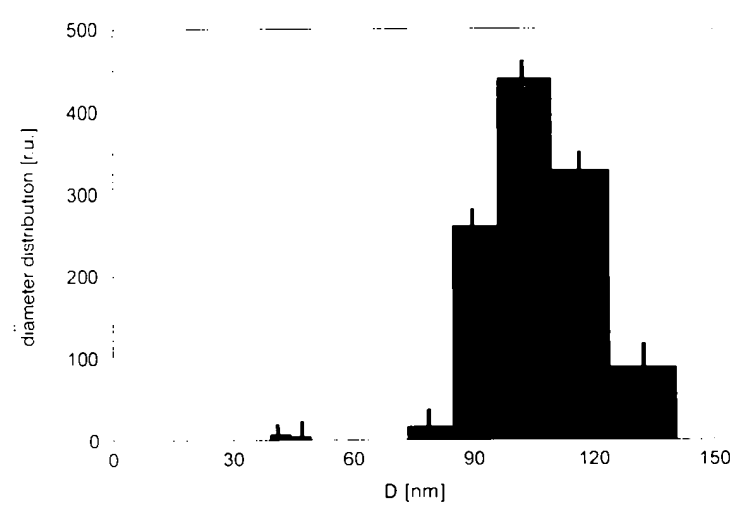

Fig. 6. Diameter distribution function of CAA nanoparticles determined from DLS using the distribution of diffusion coefficients and the Einstein equation. Grey bars: experimental data calculated with CONTIN (Provencher, 1979, 1982); black bars: error levels. The distribution corresponds to the $V^{2}$-weighted number distributions $N(D)$ calculated from SAXS

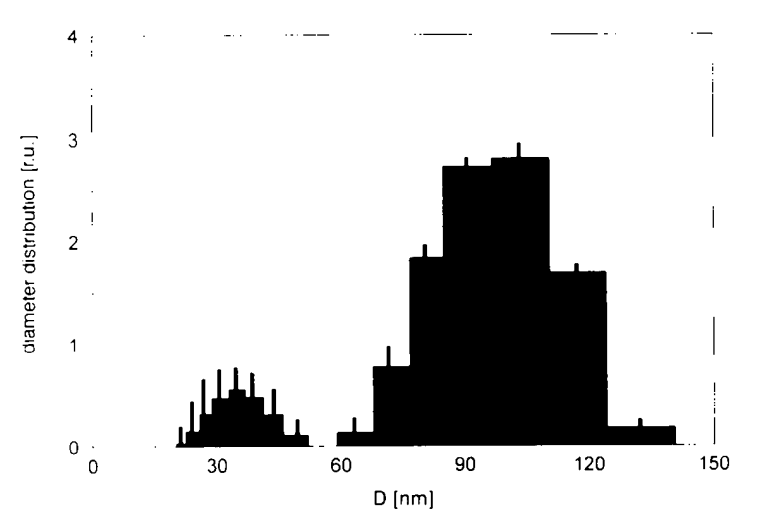

Fig. 7. Diameter distribution function of CAA nanoparticle determined from DLS using directly the distribution of diameters of hard spheres. Grey bars: experimental data calculated with CONTIN (Provencher, 1979, 1982); black bars: error levels. The distribution corresponds to the $V$-weighted number distribution $N(D)$ calculated from SAXS. about $10 \mathrm{~nm}$, either from the $V^{2}$ or the $V$-weighted size distribution, the latter being much more sensitive for small particles.

The enlarged nanoparticle dimensions of 70 to $130 \mathrm{~nm}$ in comparison with 60 to $120 \mathrm{~nm}$, obtained from DLS and X-ray scattering, respectively, can be explained by a water shell surrounding the particles and moving together with the polymer. By a fractality of the surface, where the notches act like paddle wheels, the relatively thick water shell of $10 \mathrm{~nm}$ is better explained than by polar groups (Müller, Lukowski, Kröber, Damaschun \& Dittgen, 1994) alone.

Former transmission-electron-microscopy data gave hints of a structured surface and a water-enriched surface zone of about $10 \mathrm{~nm}$ has been postulated (Zosel, 1989). No separate particles with about this diameter have been detected by Lukowski (1995). These results will be discussed in more detail elsewhere.

These observations support the fractality of the surface of the copolymer nanoparticles rather than an additional population of small spheres or inner-electron inhomogeneities. There seems to be a discrepancy concerning the $10 \mathrm{~nm}$ population between the results of direct interpretation of $\mathrm{X}$-ray scattering curves, DLS and electron miscroscopy, on the one hand, and the results of the indirect maximum-entropy method on the other. A crude model for particles with a fractal surface is used to resolve the ambiguity. A model with physical relevance has to be based on a real zero-order approximant of the fractal. This is not known in the case of the copolymer nanoparticles. Therefore, we tried a simpler approach to simulate surface rugosity. One large sphere is covered by 30 small spheres having a ninth of the radius of the large sphere (insert in Fig. 8). 10\% of the accessible surface of the large sphere is occupied by the statistically distributed small spheres. For this basic model, a diameter distribution

$$
N(D)=\exp \left[-0.5(D-81.08)^{2} / 10^{2}\right]
$$

is used for calculation of the scattered intensity using (3). As expected, the contribution of the small spheres to the scattering increases the intensity at larger angles (Fig. 8) and the curve follows the power law constant $/ s^{3.65}$. With the direct method, a particle population can be determined with a weighted mean diameter of $84.4 \mathrm{~nm}$ from the theoretical scattering curve. An additional population of small, seemingly independent, spheres with diameters of about $8 \mathrm{~nm}$ is also visible. The diameter distribution calculated with MAXENT (Fig. 8) is also bimodal. It does not show spurious oscillations but clearly shows two moieties at $8-9 \mathrm{~nm}$ and $84.7 \mathrm{~nm}$. A free Gaussian prior was taken here for the search. Both systems, a bimodal distribution consisting of large spheres with a smooth surface and small independently scattering spheres with radius a tenth of the radius of the large sphere, and a unimodal distribution of large spheres with surface-fixed 
small beads of a radius a ninth of the radius of the large sphere, are equivalent in scattering. The interaction terms between small and large spheres do not play a significant role under the chosen experimental and simulation conditions.

\section{Concluding remarks}

By using X-ray scattering, only models and particle-size distribution functions equivalent in scattering to a real object can be deduced. Direct and indirect data-handling methods are used in a complementary way. For selection of a model with high reliability, the indirect maximumentropy method MAXENT is used. As recently shown for the distance distribution, the importance of a good estimate of a prior for the particle-size distribution too has been demonstrated here by simulation. Two models for CAA nanoparticles, a pure five-population distribution of smooth hard spheres and a mixed model of a fourpopulation distribution of spheres with fractal surface were deduced from the small-angle scattering. To our knowledge, no such clearly different models have been discussed up to now that fit the experimental data within the error limits and within a restricted resolution interval. By using additional information from DLS and hints from electron microscopy, the pure multipopulation model has been called in question. Unfortunately, it was not possible to calculate directly the scattering curve for the mixed model because of lack of knowledge about the fractal geometry. But, by crude modelling of the surface rugosity by partial covering of a unimodal distribution of large spheres by smaller ones, a bimodal diameter distribution can be calculated with maximum entropy as well as with direct methods, which shows the

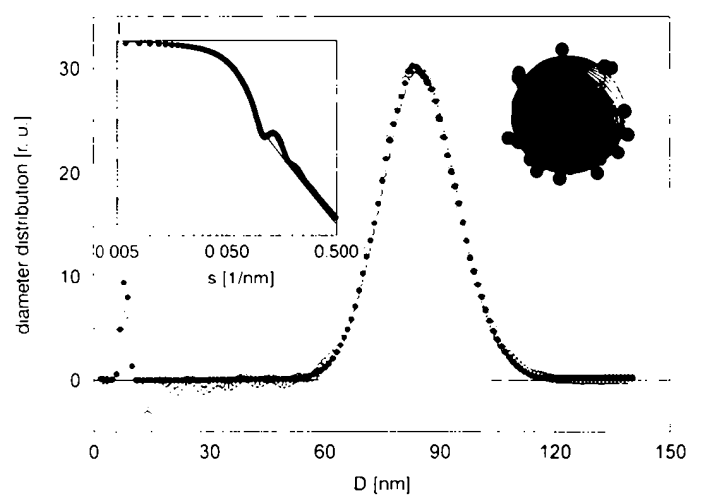

Fig. 8. Diameter distribution calculated for a Gaussian distribution of large spheres covered with small spheres of a ninth of the large radius. $10 \%$ of the accessible surface is statistically occupied by small spheres. Theoretical distribution $(\pi / 6) D^{3} \exp \left[-0.5(D-81.1)^{2}\right.$ $\left./ 10^{2}\right]$; o o o result of the direct method; - $\bullet M A X E N T$ result. Left insert: — scattering curve of the model; $\bullet \bullet$ fit by $M A X E N T$, straight line with an inclination of -3.65 (fractal dimension $d=2.35$ ). The line is shifted vertically for clarity. Right insert: model with diameters $D=81.1$ and $9.01 \mathrm{~nm}$ for the large and small spheres, respectively. small spheres as seemingly independent particle moiety. This simulation together with the experimental results from dynamic light scattering and electron microscopy renders possible a rejection of the pure multipopulation model. In reality, of course, the surface rugosity will most probably not be represented by ideally spherical beads but the irregularities will look rather like notches or bulges. Inner small inhomogeneities or a mixed model of inner fluctuations and outer rugosity cannot be excluded definitively by the applied methods but they should not play any important role because of the scattering equivalence found for model and sample and the additional information about surface rugosity from other methods. For the drug-carrier function, the detected surface fractality is relevant for further interpretation of interactions of the carrier with macromolecules, like immunoglobulins, and small surfactants or drugs, because the accessible surface is enlarged compared with smooth hard spheres corresponding to the fractal dimension.

The authors thank Udo Heinemann for helpful discussions and kind support.

\section{References}

Gast, K., Damaschun, G., Misselwitz, R. \& ZiRWER, D. (1992). Eur. Biophys. J. 21, 357-362.

Glatter, O. (1977). J. Appl. Cryst. 10, 415-421.

Glatter, O. (1980). J. Appl. Cryst. 13, 7-11.

GlatTER, O. \& KRATKY, O. (1982). Small-Angle X-ray Scattering. New York: Academic Press.

Hansen, S. \& Pedersen, J. S. (1991). J. Appl. Cryst. 24, 541-548.

JAYNES, E. T. (1983). Papers on Probability, Statistics and Statistical Physics, edited by R. D. ROSENKRANTZ. Dordrecht: Kluwer.

Jemian, P. R. \& Allen, A. J. (1994). J. Appl. Cryst. 27, 693-702.

Kreuter, J. (1983). Int. J. Pharm. 14, 43-58.

LuKOwSKI, G. (1995). PhD thesis, Ernst-Moritz-Arndt Univ., Greifswald, Germany.

LUKOWSKI, G., MÜlleR, R. H., MÜlleR, B. W. \& DitTGen, M. (1992). Int. J. Pharm. 84, 23-31.

MoOre, P. B. (1980). J. Appl. Cryst. 13, 168-175.

Morrison, J. D., CorCoran, J. D. \& LEWIS, K. E. (1992). J. Appl. Cryst. 25, 504-513.

MÜLlER, J. J. (1992). APX63, Small-Angle X-ray Scattering, pp. 1-147. Freiberg: Freiberger Präzisionsmechanik GmbH.

MÜller, J. J. \& Hansen, S. (1994). J. Appl. Cryst. 27, 257-270.

Müller, J. J., LuKowski, G., KRÖBer, R., DAMASChun, G. \& Dittgen, M. (1994). Colloid. Polym. Sci. 272, 755-769.

MüllER, R. H. (1991). Colloidal Carriers for Controlled Drug Delivery and Targeting-Modification, Characterization and in vivo Distribution. Stuttgart: Wissenschaftliche Verlagsgesellschaft; Boca Raton: CRC Press.

PFEIFER, P. (1984). Appl. Surf. Sci. 18, 146-153. 
PfEIFER, P. (1987). Preparative Chemistry Using Supported Reagents, edited by P. LASZLO, pp. 13-33. San Diego: Academic Press.

Potton, J. A., DANiell, G. J. \& Rainford, B. D. (1988). J. Appl. Cryst. 21, 663-668.

Provencher, S. W. (1979). Macromol. Chem. 180, 201-209.

Provencher, S. W. (1982). Comput. Phys. Commun. 27, 229-242.

SchmidT, P. W. (1991). J. Appl. Cryst. 24, 414-435.

SKILLING, J. (1988). Maximum-Entropy and Bayesian Methods in Science and Engineering, Vol 1, pp. 173-187. Dordrecht: Kluwer.
Steenstrup, S. (1985). Aust. J. Phys. 38, 319-327.

SVERGUN, D. I. (1992). J. Appl. Cryst. 25, 495-503.

SVErgun, D. I., SEmEnyuk, A. V. \& Feigin, L. A. (1988). Acta Cryst. A44, 244-250.

TARCHA, P. (1991). Polymers for Controlled Drug Delivery. Boca Raton/Ann Arbor/Boston: CRC Press.

Verdun, C., Courveur, P., Vranckx, H., Lenaerts, V. \& Roland, M. (1986). J. Control. Release, 3, 205-210.

Walter, G., Gerber, T. \& Kranold, R. (1983). Stud. Biophys. 97, 129-134.

Zosel, B. (1989). PhD thesis, Ernst-Moritz-Arndt Univ., Greifswald, Germany. 